\title{
Bioelectronic Medicine-Novel Way to Treat Diseases
}

\author{
Sangeeta S Chavan \\ Laboratory of Biomedical Sciences, The Feinstein Institute for Medical Research, Manhasset, New York, United States of America
}

\begin{abstract}
Online address: www.bioelecmed.org
doi: 10.15424/bioelectronmed.2014.00009
\end{abstract}

What if electronic devices can replace drugs? Future therapy using electrical signals to modulate peripheral nerves will sense and modulate neural signals to individual organs, delivering target and organ-specific effects. With recent advances in the field of molecular medicine, neurophysiology and biomedical devices, this novel way of treatment, termed "bioelectronic medicine," is within reach. Bioelectronic medicine has the potential to be superior to drugs in terms of efficacy, cost and safety because it directly modulates the natural language of the body's nervous systemselectrical impulses and action potentials.

To appreciate the full potential for bioelectronic medicine, consider that virtually all the cells in the body are directly or indirectly controlled by neural input and that peripheral neural circuits play a pivotal role in maintaining homeostasis. Physiological and molecular changes in the body are sensed and informed by the peripheral sensory neural circuits to the central nervous system, which in turn activates a motor response reinstating the homeostasis in the target tissue. Neural signals mediating information about the biological activity of each cell are trans- mitted as electric information in the form of action potentials traveling along axonal fibers, to and from the innervated tissues and organs. By use of bioelectronic medicine, it is now possible to modulate these electric signals. Miniaturized devices can be implanted at selective nerve fibers to either stimulate or block neural activities as a therapeutic modality to treat a broad spectrum of conditions. It is interesting to consider that by converging neurophysiology with data analysis and disease biology, it will be feasible to develop bioelectronic devices that can record and analyze neural and physiological data in real time and modulate the neural electric input to the target organs.

In this inaugural issue of Bioelectronic Medicine, we report on the Centricity Series symposium Bioelectronic Medicine: A next generation of treatment, which was held in March 2014 at the Feinstein Institute for Medical Research in New York. At the meeting, more than 100 investigators gathered to share the latest advances in the field of bioelectronic medicine, and the significant progress in this area is highlighted in this introductory issue of Bioelectronic Medicine. The

Address correspondence to Sangeeta S Chavan, The Feinstein Institute for Medical Research, Laboratory of Biomedical Sciences, 350 Community Drive, Manhasset, NY 11030. Phone: 516-562-21 18; Fax: 516-562-2356; E-mail: schavan@nshs.edu.

Submitted October 23, 2014; Accepted for publication October 23, 2014; Published Online (www.bioelecmed.org) December 2, 2014.

\section{The Feinstein Institute for Medical Research

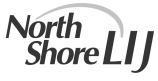
Empowering Imagination. Pioneering Discovery. 
ment of certain brain disorders. However, the exact site for optimal stimulation is still unknown. Here, a network analysis strategy using graph theory may help in the identification of diseaserelated brain networks and optimization of target identification.

Larry Miller and Anil Vegesna review another promising area for bioelectronic medicine: targeting gastrointestinal disorders, specifically sphincteric dysfunctions. Sphincters control passage of solids, liquids and gases in the gastrointestinal tract; and defective sphincters lead to gastrointestinal disorders. Bioelectronic devices that deliver electric current to the sphincteric muscle or to the nerves innervating the muscle modulating the sphincter contractibility may be utilized to target gastrointestinal dysfunctions.

In their review, Jason Fritz and Jared Huston discuss application of bioelectronic medicine to regulate surgical and traumatic hemorrhage through a novel mechanism termed the "neural tourniquet." Bioelectronic devices that activate vagus nerve-mediated pathways significantly improve hemostasis in traumatic hemorrhage models and thus demonstrate a prime example of how bioelectronic devices can help fight one of the long-standing problems in human history-uncontrolled bleeding.

The inflammatory reflex, a prototypical neural circuit controlling immunity, comprises afferent and efferent neurons that travel in the vagus nerve to regulate systemic immune responses; this reflex forms the topic of the review by Peder Olofsson. In this reflex circuit, afferent vagus nerve fibers report inflammatory events to the brainstem, which elicits regulating motor signals returning to the periphery. By using bioelectronic devices, it is now possible to modulate the inflammatory reflex to treat inflammatory diseases.

Finally, Yaakov Levine and colleagues review applications of modulating the inflammatory reflex in experimental models of inflammatory diseases. Extensive studies with animal models indicate that stimulation of inflammatory reflex using implantable bioelectronic devices is a feasible therapeutic approach for the treatment of rheumatoid arthritis and inflammatory bowel disease. On the basis of this knowledge, implanted vagus nerve stimulators are now being used in exploratory clinical trials to treat rheumatoid arthritis and colitis.

It seems clear that bioelectronic medicine will be a prolific area of research in the coming years. Targeting electrical impulses of peripheral neurons using bioelectronic miniaturized devices will soon become a mainstay of medical treatment.

\section{DISCLOSURE}

The author declares that she has no competing interests as defined by Bioelectronic Medicine, or other interests that might be perceived to influence the results and discussion reported in this paper.

Cite this article as: Chavan SS. (2014) Bioelectronic medicine-novel way to treat diseases. Bioelectron. Med. 1:2-3. 